\title{
Wissenschaft und Magie
}

\author{
Ethnologische und wahrnehmungspsychologische Motive \\ in Ludwik Flecks Epistemologie
}

Sylwia Werner

Science and Magic. Motives from Ethnology and the Psychology of Perception in the Epistemology of Ludwik Fleck

Fleck's social theory of science refers to many ethnological examples in order to explain how collective thinking and acting constructs certain systems of belief and knowing. According to Fleck, scientific concepts and practices are comparable with magic terms and ceremonies. This essay aims to identify the ethnological sources that Fleck's epistemology is using. By confronting them with other relativistic theories that were circulating in Lemberg during the interwar period, the originality of Fleck's own position can be contextualized and explained as well.

Keywords: Ludwik Fleck, ethnology, perception, cultural relativity, Bronisław Malinowski, circulation of knowledge, thought-style, thought-collective, Lévy-Bruhl

Schlüsse/worte: Ludwik Fleck, Ethnologie, Wahrnehmung, Kulturrelativismus, Bronisław Malinowski, Zirkulation von Wissen, Denkstil, Denkkollektiv, Lévy-Bruhl

Für die Geschichte der wissenschaftlichen Moderne sind neben den Metropolen Wien, Berlin oder Paris auch viele osteuropäische Zentren von großer Bedeutung, die jedoch ungleich weniger erforscht sind. So gab es auch beispielsweise in Lemberg eine quer durch die Disziplinen gehende rege Entwicklung verschiedener epistemologischer Konzeptionen, die in ihrer Radikalität und Modernität im westlichen Europa ihresgleichen suchten. $\mathrm{Zu}$ den wichtigsten von ihnen gehört die eine soziologische und kulturalistische Bestimmung von Tatsachen postulierende Wissenschaftstheorie Ludwik Flecks. Im Folgenden werde ich Flecks Epistemologie auf Bezüge zur Ethnologie und Wahrnehmungstheorie untersuchen und dabei die Verbindungslinien $\mathrm{zu}$ den in Lemberg kursierenden und miteinander konkurrierenden Konzeptionen, wie etwa denen von Jan Dembowski, Jakob Frostig, Bronisław Malinowski, Izydora Dąmbska und Tadeusz Bilikiewicz aufzeigen. Ferner soll beleuchtet werden, inwiefern die antiindividualistische und antiobjektivistische Position Flecks sich mit westeuropäischen Theorien von Lucien Lévy-Bruhl oder Wilhelm Jerusalem auseinandersetzte und dabei 
über diese hinausging. Hierbei stellt sich die Frage, inwiefern sich Flecks Epistemologie einer bestimmten wissenschaftlichen Tradition zuordnen lässt und wie sie sich zu den seinerzeit dominierenden Theorien und Schulen stellt. Das Ziel des vorliegenden Beitrags ist es zu überprüfen, ob und wenn ja, aus welchen ethnologischen und soziologischen Quellen Flecks relativistische Wahrnehmungskonzeption gespeist wurde und wie sie von ihm für sein Konzept der sozialen und kulturellen Bedingtheit von Wissenschaft umgeformt wurden. Dabei geht es weniger darum, Einflussbeziehungen aufzuzeigen, vielmehr sollen intertextuelle Bezüge und inhaltliche Korrespondenzen herausgearbeitet werden.

\section{Der Entwurf einer relativistischen Epistemologie im Lichte der medizinischen Praxis}

Fleck entwickelte seine „Lehre vom Denkstil und Denkkollektiv“ in der Zwischenkriegszeit in Lemberg und damit an einem Ort, wo auf engem Raum Wissenschaftler, Künstler und Philosophen an gemeinsamen Denk- und Schreibweisen partizipierten (Werner 2011). Womöglich war es diese enge Verbindung zwischen wissenschaftlichen und künstlerischen Konzepten, die es Fleck erlaubte, eine Epistemologie zu entwerfen, in der Methoden zum Einsatz kamen, die man bislang für die Geistes- und Sozialwissenschaften reserviert hatte. Damit trat er in die Opposition zu der in Lemberg um Kazimierz Twardowski gruppierten Schule der philosophischen Logik, die in Anlehnung an die Programmschriften des Wiener Kreises (Stadler 1997), die Position des logischen Empirismus vertrat und sich zum Ziel setzte, durch eine rationale Rekonstruktion der Logik der Forschung kulturelle Faktoren, die die Forschungspraxis kontaminieren, nachträglich herauszufiltern. Wie Roman Ingarden bilanzierte, führte „der überwiegende Einfluß der österreichischen Schule" - gemeint ist der logische Positivismus - zu einem

\footnotetext{
fast völligem Ausschluß jener philosophischen Richtungen, die um Jahrhundertwende auf verschiedenen Wegen eine Renaissance der Philosophie suchten, wie z.B. der Neukantianismus in seinen verschiedenen Gestaltungen, die Phänomenologie, Bergson, der Pragmatismus, Dilthey und die sogenannte geisteswissenschaftliche Philosophie. [...] Keine von diesen damals sich mächtig entwickelnden philosophischen Richtungen konnte in Lemberg Fuß fassen. (Ingarden 1937: 226 f.)
}

Fleck befand sich also in einer schwierigen Ausgangsposition, zum einen war er kein Philosoph, sondern Mediziner, zum andern fügten sich seine Ansichten nicht in die damaligen wissenschaftlichen Diskussionen und in die Argumentationskultur des vorherrschenden logischen Empirismus in Polen und über Polen hinaus. Dies erklärt zum Teil, warum Flecks Epistemologie lange in der 
Philosophie ohne Widerhall blieb (Wegmarshaus 2007: 51). Andererseits gab es die von Ingarden erwähnten anderen mächtigen Strömungen in Europa und mit ihnen wuchs der Druck, eine Theorie zu entwickeln, die auch die Entwicklungen in den Geistes- und Sozialwissenschaften nicht einfach ignoriert oder methodisch ausgrenzt, sondern in die eigene Theorie aufnimmt. Die neuen Erkenntnisse aus Soziologie, Ethnologie und vergleichenden Kulturtheorien nahm Fleck insofern auf, als er eine Theorie pluralistischer Denkstile entwickelte und hierdurch die sehr verschiedenen Ansätze seiner Zeit, die Positionen von Durkheim, Lévy-Bruhl, Bergson und Malinowski, aber auch die Wissenschaftstheorie Carnaps zusammendenken konnte. Fleck offerierte also im damaligen Kampf um die richtige wissenschaftliche Weltauslegung eine Metatheorie, die die Koexistenz verschiedener Denkstile annahm und dabei weder darauf aus war, einer Position zum Sieg zu verhelfen noch, die Gegensätze durch Konzeptionen des kultur- beziehungsweise denkstilübergreifenden Verstehens zu mildern.

Fleck musste daher zunächst außerhalb der philosophischen und wissenschaftstheoretischen Zirkel Lembergs und anhand seines eigenen Spezialgebiets in der Medizin versuchen, seine Problemstellung gegen die vorherrschenden Theorien ins Blickfeld zu rücken. 1926 präsentierte er in der "Gesellschaft der Freunde der Medizingeschichte" seine ersten wissenschaftstheoretischen Erkenntnisse in einem Referat unter dem Titel „Über einige spezifische Merkmale des ärztlichen Denkens“. Hierbei beschrieb er die Medizin als eine prinzipiell unlogische, weil auf irrationalen Annahmen fußende Disziplin, die beständig Gesetze für nicht gesetzesmäßige Phänomene aufstelle und sich dabei permanent selbst szientistisch mißverstünde (Zittel 2007: 444). Die auf induktiven Verfahren beruhenden medizinischen Diagnosen seien „wissenschaftliche Fiktion[en]“ (Fleck 2011 [1927]: 47), die nicht durch neutrale Beobachtung, sondern vielmehr durch intuitives Ahnen hervorgebracht werden. Dem zufolge könnten medizinische Phänomene nicht logisch rekonstruiert werden, da sie stets aus unklaren und nie exakt zu definierenden Ursachen resultierten. Fleck konstatiert:

In der Medizin tritt der in seiner Art einzigartige Umstand auf, daß je schlechter ein Arzt ist, um so „logischer” seine Therapie ist. Denn scheinbar läßt sich in der Medizin fast alles als Beweis angeben, so daß wir bisher tatsächlich fast nichts aufgeklärt haben. (Ebd.: 45)

Auf der Basis einer nüchternen Beschreibung der medizinischen Praxis entfaltet Fleck seine Kritik an der allzu idealistischen medizinischen Wissenschaftstheorie. Die medizinische Praxis sei, gerade weil in ihr Intuition und Erfahrung dominieren, eine durch und durch kulturell geprägte Praxis. Sie gründe nicht auf objektiven Befunden, sondern auf traditionellen Riten, die diese Befunde erst objektiv erscheinen lassen. Fleck beschreibt das Spezifische der Medizin, als verfahre sie (allerdings mit Ausnahme des Bezugs auf 
statistische Methoden) noch weitgehend wie Paracelsus, als würden in ihr immer noch die gleichen Prinzipien gelten, die für die Praktiken der Renaissancemagier oder Wunderheiler charakteristisch waren und die man überwunden $\mathrm{zu}$ haben glaubte. Doch Fortschrittsvorstellungen in der Medizin, wie sie etwa vom Paracelsus-Kenner Karl Sudhoff vertreten wurden (Fleck 2011 [1935]: 242), ersetzt Fleck hier bereits durch eine Konzeption, die lediglich einen Wechsel verschiedener medizinischer Kulturen annimmt. Entsprechend wird er von diesem Zeitpunkt an immer stärker relativistische Konzeptionen aus der Ethnologie, Psychologie, Wissenssoziologie und Umweltbiologie rezipieren und nicht nur die Medizin, sondern auch andere Wissenschaften mit ethnologischen und psychologischen Termini beschreiben.

Die von Fleck beobachtete Nähe der Medizin zu magischen Praktiken greift in Lemberg der renommierte Biologe Jan Dembowski (1889-1963) auf. In seinem 1927 erschienenen Text „Über die Poesie der Wissenschaft“ betont er die „schöpferische Phantasie“ (Dembowski 1927: 2) des Forschers, die den Beobachtungsprozess beziehungsweise die Wahrnehmung von Experimenten begleite und schließlich zur Formulierung von neuen wissenschaftlichen Gesetzen führe:

Fern von der Welt und ihrer lärmenden Angelegenheiten gibt sich der Forscher seinen Phantasien hin und seine Einbildungskraft zeichnet ihm noch undeutliche, vernebelte Bilder. Erst beginnt eine mühselige Sortierungs-, Verschiebungs- und Annäherungsarbeit an mitgebrachten Tatsachen, in denen der Forscher nach der Widerspiegelung seiner Phantasien sucht. Über der dichten Masse zahlreicher Phänomene schwebt eine Vision, eine unergreifbare, nicht-materielle Gestalt, deren Umriß wir eher erahnen als sehen. Es kommen neue Tatsachen, neue Zusammenhänge und die phantastische Gestalt hebt sich immer deutlicher vom Hintergrund ab. Plötzlich passiert ein Wunder: Die Gestalt bekommt Leben! Es wurde ein neues wissenschaftliches Gesetz geboren. (Ebd.: 4 f. Übersetzung SW)

Wie Fleck erkennt auch Dembowski, dass die Geschichte der (biologischen) Wissenschaft einen ästhetischen Ursprung hat, wobei Phantasie und Vision als Geburtshelfer fungieren, und mehr noch: Dembowski scheint einige von Flecks späteren Beobachtungen zum Wahrnehmen von Gestalten und der wundersamen Epiphanie von gesetzesförmigen Tatsachen in einer ebenfalls noch etwas unklaren Vision vorwegzunehmen. In seinem 1946 erschienenen Buch Tierpsychologie verwendet er Begriffe wie etwa „Transposition“, „positive“ und „,negative Gestalt“, aber auch Bildexempel für die Transposition von Buchstaben und Worten, die Fleck ein Jahr später in „Schauen, Sehen, Wissen", benutzt, allerdings zu einem anderen Zweck (vgl. dazu den Beitrag von Claus Zittel in diesem Heft). Denn während Dembowski auf das Gestaltprinzip zurückgreift, um die Wahrnehmung von Fischen, Bienen oder Affen auf physiologische und motorische Funktionen zu untersuchen, bedient sich Fleck der gleichen Wahrnehmungsexempel, um die kulturelle Bedingtheit 
des kollektiven Sehens von Ganzheiten zu demonstrieren. Zudem beobachtet Dembowski, dass die durch die Tiere wahrgenommenen Dinge in ihrem Nervensystem so fest verankert bleiben, dass sie automatisch wiedererkannt werden, das heißt wie bedingte Reflexe beziehungsweise angeborene Reize fungieren: So lernt der Fisch zum Beispiel den Buchstaben „L“ vom Buchstaben „R“ zu unterscheiden, wenn mit dem Anblick von „L“ stets eine Futterbelohnung, mit dem Anblick von „R“ hingegen eine Strafe verbunden ist. In dem Moment, in dem der bedingte Reflex fixiert ist, wird der Buchstabe „L“ $\mathrm{zu}$ einer Gestalt mitsamt aller ihrer Transpositionen. Fleck gibt dieser Argumentation eine soziologisch-kulturalistische Wendung und schmiedet aus ihr eine antiindividualistische Wahrnehmungstheorie:

Wir schauen mit den eigenen Augen, aber wir sehen mit den Augen des Kollektivs Gestalten, deren Sinn und Bereich zulässiger Transpositionen das Kollektiv geschaffen hat. Wir sind geneigt, sie zu vervollständigen, im positiven und negativen Sinn, d.h. wir sehen nicht, daß gewisse Elemente fehlen, und wir erblinden gegenüber überflüssigen Zusätzen. (Fleck 2011 [1947]: 400)

Ein isoliertes Forschergenie existiert Fleck zufolge nicht. Bereits in den 1930er Jahren hatte er dargelegt, dass Entdeckungen nicht von einzelnen Individuen gemacht werden, sondern von denkstilgebundenen Gruppen. Folglich gebe es keinen freien neutralen Denk-Raum, in dem verschiedene Denkweisen ausprobiert werden könnten, sondern denkstildurchwirkte Räume, in denen ein „Denkzwang“ herrsche, der durch rituelle Praktiken und unhinterfragt übernommene Einstellungen aus der Tradition ausgeübt werde. Forscher werden somit bereits in ihrer Ausbildung gleichsam dressiert und ihre Erkenntnis von vornherein auf bestimmte Erkenntnisinhalte ausgerichtet (Zittel 2011):

In der wissenschaftlichen Beobachtung gibt es die bestimmte Bereitschaft zu gewissen Beobachtungen, sie ist vor allem durch eine gewisse Schulung, durch eine gewisse wissenschaftliche Tradition hervorgerufen. (Fleck 2011 [1935]: 213)

Und weiter:

Der Forscher hat kein Bewußtsein der Auswahl, im Gegenteil, die Auswahl drängt sich ihm direkt und bindend auf, indem sie aus seiner Denkstimmung, aus dem Komplex seiner geistigen Bereitschaften, aus seinen Denkgewohnheiten hervorgeht - kurz gesagt: aus dem, was ich Denkstil nenne. (Ebd.: 219)

An die Stelle eines Individuums tritt folglich eine „Gemeinschaft von Menschen mit gemeinsamem Denkstil” (ebd.: 226), das heißt ein „Denkkollektiv”, das über ein „Bereitsein für solches und nicht anderes Sehen und Handeln" (Fleck 1980 [1935]: 85) verfügt. Es liegt auf der Hand, dass sich die so beschriebenen kollektiven Praktiken für soziologische und ethnologische Betrachtungen anbieten und es ist interessant zu verfolgen, ab wann Fleck Modelle und Beispiele aus der Ethnologie aufgreift, um mit ihnen seine Argumentation zu veranschaulichen und zu verstärken. 


\section{Woran konnte sich Fleck seinerzeit orientieren?}

Ein systematischer Vergleich mit einigen in Lemberg zirkulierenden und theoretisch verwandten Theorien soll erhellen, inwieweit Flecks Epistemologie in die ethnologischen und wahrnehmungstheoretischen Diskurse eingebettet werden kann.

Einen der Belege dafür, dass Flecks Konzept im Kontext der Lemberger Wissenschaftstheorie zu verorten ist, liefert das Buch des Psychiaters Jakob Frostig (1896-1959) das 1929 unter dem Titel Das schizophrene Denken. Phänomenologische Studie zum Problem der widersinnigen Sätze erschien (vgl. Fehr 2011). Frostig stellt hier nicht einfach klinische Analysen von Fallgeschichten vor, sondern versucht mit Hilfe der Phänomenologie eine neue Beschreibungsform psychischer Störungen einzuführen und wahrnehmungstheoretisch auszudeuten. ${ }^{1}$ Dabei geht er, und Fleck wird ihm später folgen, von kollektiv organisierten, sozialen Gruppen aus, die durch eine gemeinsame "Gesinnung“ verbunden seien und die aufgrund der von ihnen entwickelten sozialen Interaktionen unterschiedliche Wirklichkeiten gestalteten [sic!]:

Unter „Wirklichkeit” werden wir künftighin nicht jene Wirklichkeit verstehen, die der europäischen Gruppe im Wege einer wissenschaftlichen Erkenntnis sich als „absolut wahr" vorstellt. [...] Wir werden also relativ von vielen Wirklichkeiten sprechen und werden in einer Beschreibung die phänomenologischen Eigenschaften einer Wirklichkeit, wie sie sich etwa die Botokuden denken, in derselben Art aufzudecken versuchen, wie etwa jene Wirklichkeit, wie sie sich dem Durchschnittseuropäer, oder gar der Gesinnungsgruppe der Astrophysiker vorstellt. Wirklichkeit heißt also in unserer Untersuchung - die Wirklichkeit, wie sie einer Gruppe gegeben ist. (Frostig 1929: 24, Fußnote 1)

Diese kulturrelativistisch-ethnologische Definition der Wirklichkeit gelte allerdings nicht für das Sprach- und Denksystem eines Schizophrenen, der Frostig zufolge - unfähig sei, soziale Interaktionen zu entwickeln. Da seine Aussagen ,widersinnig” seien, das heißt keinen Bezug auf kollektive Sinnzuschreibungen haben, bliebe er aus dem assoziativen Denken einer sozialen Gruppe ausgesperrt. Das Motiv eines sich der Wirklichkeit entziehenden Schizophrenen greift Fleck in seinem noch im gleichen Jahr erschienenen Text „Zur Krise der ,Wirklichkeit” auf. Am Beispiel eines „widersinnigen Satzes” argumentiert er mit Frostig, dass auch die Wirklichkeit eines Geisteskraken nicht frei von kulturellen Einflüssen sei:

Auch der Schizophrene, dessen asozialer Augenblickswirklichkeit Aussprüche wie „1-2 - 3 das ist Apotheke, das ist Buchs, Rio de Janeiro" entspringen, gebraucht sozial entstandene Begriffe. Doch seine Wirklichkeit bleibt für andere - und wahrscheinlich auch für ihn selbst im nächsten Augenblicke - verschlossen. (Fleck 2011 [1929]: 54 f.)

Fleck benutzt Frostigs kulturrelativistische Konzeption, um auch in der labororientierten Praxis die Wahrnehmung medizinischer Phänomene 
theoretisch einzuholen. Hierbei bedient er sich des Begriffs des „Widerstandsavisos“, mit dem er den Moment der Wiedererkennung im Erkenntnisprozess meint. Die „Willkürlichkeit des Denkens” (Fleck 1980 [1935]: 132), das „schöpferische Chaos der Gedanken” (Fleck 2011 [1935]: 227) werden dabei gehemmt und der Wahrnehmungsapparat wird auf ein bestimmtes Muster gerichtet: „So entsteht die Tatsache: Zuerst ein Widerstandsaviso im chaotischen anfänglichen Denken, dann ein bestimmter Denkzwang, schließlich eine unmittelbar wahrzunehmende Gestalt" (Fleck 1980 [1935]: 124).

Ein Aviso heißt ja zunächst nur ein Anzeichen, in der Medizin ein Symptom, das eine Krankheit anzeigt und dabei auf eine reale Ursache hinter dem Phänomen verweist. Doch Fleck definiert Aviso nicht als ein objektives Erkenntnismittel, sondern als eins, das denkstilgemäß determiniert ist. Dies gilt auch für den von Frostig zuvor gebrauchten Begriff „,intentionales Aviso“, das die Erkenntnis auf ein „ideales Gebilde” (Frostig 1929: 19) lenkt und das als Vorbildkonzept für Flecks so eigentümlich scheinende Rede von einem „Widerstandsaviso“ anzusehen ist: „Alles andere wird außer acht gelassen, von allem Anderen sind wir bemüht zu abstrahieren, alles andere wird in ,Klammer gesetzt" " (ebd.). Das Erkennen wird somit bei Frostig immer intentional, also auf den Denkakt und seine Gerichtetheit bezogen und nicht auf einen objektiven Gegenstand außerhalb des Denkens. ${ }^{2}$

Eine andere Quelle, auf die sich Fleck bei der Entwicklung seiner Epistemologie stützen konnte, ist die kulturanthropologische Theorie von Bronisław Malinowski (1884-1942). Obgleich sich zwischen Malinowski und Fleck kein direkter Bezug weder persönlicher Art noch über Textverweise rekonstruieren lässt, kam es möglicherweise doch über den gemeinsamen Lemberger Freundeskreis, in dem die neuen Ideen und Methoden zirkulierten, zu einem mittelbaren gedanklichen Austausch. Denn Flecks Wissenschaftstheorie weist viele inhaltliche Korrespondenzen mit Malinowskis bahnbrechendem „Argonautenbuch" (Malinowski 1922) sowie seinen späteren theoretischen Schriften auf. In seiner aus der teilnehmenden Beobachtung entwickelten Funktionaltheorie, die auf der Überzeugung gründet, dass die Kultur fremder Völker nicht aus der Distanz (vom Schiffsdeck oder dem heimischen Lehnstuhl aus), sondern direkt durch die unmittelbare Konfrontation zu erforschen sei, fordert Malinowski, die Denk- und Verhaltensweisen der Menschen immer in Relation zum gegebenen Kultursystem zu untersuchen. In der neueren Forschung wird Malinowskis Vorgehensweise als Veränderung des damaligen wissenschaftlichen Weltbildes, ja als Begründung eines neuen „methodologischen Paradigmas” (Stocking 1978, Stagl 1993) in der Ethnologie angesehen. Der Paradigmen-Begriff erscheint jedoch als zu grob, um die damaligen Veränderungen in der Wissenschaftstheorie zu beschreiben, hier wären Binnendifferenzierungen angebracht. Denn wie Malinowski geht auch Fleck im Sinne der teilnehmenden Beobachtung vor beziehungsweise sieht er sich als Teil der Laborpraxis und beeinflusst diese (Fleck 2011 [1946]). Im 
Unterschied zu Malinowski ist sich Fleck jedoch im Klaren, dass durch den Akt der teilnehmenden Beobachtung das Beobachtungsresultat kontaminiert wird und hierbei die eigenen kulturellen Prägungen weitgehend unerkannt mit ins Spiel kommen. Hier profitiert Fleck von seiner Rezeption der wissenschaftstheoretischen Diskussion um die Rolle der Beobachtung im Bereich der Quantenphysik (Bohr 1928). Auf einer abstrakteren Ebene weist Flecks kulturalistische Epistemologie jedoch eine Nähe $\mathrm{zu}$ Malinowskis Untersuchungsmodell sozialer Gruppen beziehungsweise (Kultur)Gemeinschaften auf. Denn auch Malinowskis Auffassung nach gibt es keine anthropologische Wesensausstattung des Menschen, die der Kultur vorgängig wäre, weshalb kein Impuls, kein Reflex, aber auch „kein Gedanke und kein Gegenstand isoliert von einem Kultursystem erfaßt werden kann". ${ }^{3}$ Die kulturelle Durchdringung erstrecke sich in die Physiologie, Zwänge werden körperlich einverleibt, der ganze Organismus werde somit in ein kulturbedingtes System eingeübt und es sei eben der ,innere Zwang” (inner constraint), der ihn in das gegebene Normen- und Ordnungssystem einbindet. ${ }^{4}$ Malinowski spricht hier noch von einer Machtform, die einen regulativen Charakter hat. Fleck versteht unter „Denkzwang” eine mehr oder weniger bewusste Form der Denknotwendigkeit, etwas nur noch so und nicht mehr anders denken und sehen zu können. Diesem „Denkzwang” ging eine Phase des sozialen Drucks voraus, in der ein Einzelner in eine Gruppe eingeführt und gleichsam initiiert wird. Diese Depotenzierung des Einzelnen weist ebenfalls eine erstaunliche Nähe zu Malinowskis kollektivistischer Position auf. Denn auch er untersucht nicht einzelne Individuen oder Handlungen, sondern fragt nach ihren Funktionen, die sie im sozialen Gefüge haben. Er beobachtet, dass Gewohnheiten innerhalb von bestimmten Gruppen normativ werden und sich so in der gegebenen Kultur verankern. Analog zum Denkstil kennt er also gruppenspezifische Routinen, Denk- und Handlungsweisen, die zu einer gruppeninternen Solidarität führen, innerhalb der Gruppe tradiert werden, und die sich nicht einfach von einer Gruppe auf die andere übertragen lassen:

Were one to look more closely at any particular culture, every activity would be found to be related to some organization or other. In each we would find a group cooperating, linked by common interests and a purpose. Members of such of group or institution [...] obey prescribed norms of conduct and are trained in particular skills. (Malinowski 1941: 185)

Eine solche sozial organisierte Gruppe erfüllt bei Malinowski dieselbe Funktion wie bei Fleck das Denkkollektiv. Sie ist der eigentliche Akteur in einem Erkenntnisprozess. Die Kategorie der individuellen Autorschaft hebt somit auch Malinowski auf. Ihm zufolge sind wissenschaftliche Erfindungen als kollektive Errungenschaften einer Zivilisation zu verstehen. In seinem 1926 erschienenen Text „The Life of Culture” heißt es: 
The fact is that each invention is arrived at piece-meal, by infinitely many, infinitely small steps, a process in which it is impossible to assign a precise share to any one worker or still less to connect a definite object and a definite idea with a single contribution. [...] The real pathway of ideas and achievements goes through hundreds and thousands of humbler workers and laboratory mechanics, the mathematicians and engineers who jointly made the final success possible. Thus the invention of the wireless can be treated as a single and singular event and ascribed to one man or another only after its nature has been completely misconceived. This is quite legitimate from the point of view or the patent office but quite erroneous for the science of culture. (Malinowski 1926: 179)

Das was Malinowski als Aufgabe einer Kulturwissenschaft beschrieb, nämlich die Analyse kollektiver Handlungen, wurde bereits lange zuvor, etwa von den Soziologen Ludwig Gumplowicz (Gumplowicz 1885) und Émile Durkheim (Durkheim 1976 [1898]) gefordert (Egloff 2007, siehe auch die Beiträge von Eva Johach und Rainer Egloff in diesem Heft). Fleck zitiert zwar Gumplowicz, orientiert sich aber mehr an den neueren wissenssoziologischen Theorien. In der Entstehung und Entwicklung einer wissenschaftlichen Tatsache (Fleck 1980 [1935]) fordert er eine vergleichende Denkstilforschung, die verschiedene Kultursysteme zueinander in Beziehung setzt und verwandte Begriffe miteinander vergleicht. Hierbei greift er die Konzeption von Lucien Lévy-Bruhl auf (Griesecke 2009), einem Schüler von Durkheim, der in seinem Buch Das Denken der Naturvölker (Lévy-Bruhl: 1926) verschiedene Denkweisen der „primitiven” Menschen miteinander vergleicht und dabei unser logisches Denken zum prälogischen in Beziehung setzt. ${ }^{5}$ Obwohl Lévy-Bruhl aufzuzeigen versucht, dass das Studium der Kollektivvorstellungen der Urvölker dazu genutzt werden könne, auch in unserer „,modernen” Wissenschaft den Einfluss von Kollektivvorstellungen zu erkennen, nimmt er schließlich doch eine qualitative Trennung zwischen einer modernen und einer primitiven Gesellschaft vor und setzt voraus, dass das eigene wissenschaftliche Denken dem vermeintlich primitiven überlegen ist. Fleck geht hier weit darüber hinaus und zeigt anhand der Geschichte der Wassermann-Reaktion sowie der eigenen Forschungspraxis auf, dass auch das Denken eines „,modernen” Forscherkollektivs ebenso unaufhebbar von kulturellen Praktiken und rituellen Handlungen abhänge wie das der „Primitiven”. Auch unsere Wissenschaft sei durch den Zwang der Tradition sowie gruppendynamische Prozesse, eine spezifische „Denkstimmung“ (Fleck 2011 [1934]: 198), einen „Denkzauber“ (Fleck 2011 [1936]: 285) und eine „Denksolidarität“ (Fleck 1980 [1935]: 140) vollständig determiniert. Eine Hierarchisierung oder Trennung von präwissenschaftlich und wissenschaftlich gäbe es nicht absolut, sondern nur innerhalb der Sicht eines gegebenen Denkstils.

Diese Auffassung bekräftigt Fleck noch einmal in der Kritik am Wiener Philosophen Wilhelm Jerusalem, der in seiner 1924 erschienenen Schrift „Die soziologische Bedingtheit des Denkens und der Denkformen” (Jerusalem 
1924) einen Fortschritt beziehungsweise einen Übergang vom primitiven zum modernen Denken beschreibt. Der primitive Mensch lebe in sozialer Gebundenheit und sei vollkommen von „Kollektivvorstellungen” (Lévy-Bruhl 1926) bestimmt. In seiner ersten Entwicklungsphase glaube er an Dämonen und Geister, und sei noch unfähig, objektiv zu denken. Langsam entwickle er sich zu einer selbständigen Persönlichkeit und befreie sich allmählich von seinen „Kollektivvorstellungen”. Erreicht er diese Stufe, sei er imstande, theoretisch zu denken und objektive Vorstellungen zu projizieren. Fleck lehnt diese Überzeugung ab: Zwar kennt er den Wandel von Denkstilen, doch diese werden nicht als objektive Fortschritte angesehen, sondern der Eindruck des Fortschritts ist wiederum denkstilgebunden. Einen solchen Prozess der gegenseitigen Bestärkung innerhalb einer Gruppe nennt Jerusalem eine „soziale Verdichtung” (Jerusalem 1924: 192) - ein Begriff, den Fleck übernehmen wird, um die Herausbildung eines gemeinsamen „Denkstils” durch einen regelmäßigen „Denkverkehr” zu beschreiben. Sowohl für Jerusalem als auch für Fleck ist jene „soziale Verdichtung” (Fleck 1980 [1935]: 64) eine Voraussetzung für die wissenschaftliche Erkenntnis, doch was Fleck bei Jerusalem moniert, ist dessen Überzeugung, es gebe die Objektivität wissenschaftlicher Tatsachen. In Europa habe sich im Unterschied zu anderen Kulturkreisen doch eine sukzessive Befreiung oder Reinigung der Wissenschaft von außerwissenschaftlichen Faktoren ereignet und schreite weiter fort. Sowohl Lévy-Bruhl als auch Jerusalem begehen somit in Flecks Augen - „so fördernd ihre Gedanken sind - einen charakteristischen Fehler: sie haben allzu großen Respekt, eine Art religiöser Hochachtung vor naturwissenschaftlichen Tatsachen" (ebd.: 65). Auch unsere Wissenschaft sei von mannigfachen mythisch-historischen Elementen, wie institutionellen Einweihungsritualen, Zeremonien und Aufnahmesakramenten bestimmt. Fleck stellt hier nun klar den Bezug zur Ethnologie her:

[Die] Einweihung in einen Denkstil, also auch die Einweihung in eine Wissenschaft sind erkenntnistheoretisch jenen Einweihungen analog, die wir aus der Ethnologie und Kulturgeschichte kennen. Sie wirken nicht nur formell: der heilige Geist senkt sich auf den Neuling herab und bis jetzt Unsichtbares wird sichtbar. Dies ist die Wirkung der Aneignung eines Denkstils. (Ebd.: 137)

\section{Kontroversen um Flecks Denkstil-Begriff}

Mit der Positionierung des Denkstil-Begriffs in der Ethnologie provoziert Fleck in Lemberg Widerspruch, vor allem seitens der konservativen, sich der Semantik und Logik widmenden Lemberg-Warschau-Schule. Ihre Vertreterin - die Philosophin Izydora Dąmbska (1904-1983) attackierte 1937 Flecks kulturalistische Auffassung der Wissenschaft, indem sie darauf bestand, dass 
die Wissenschaft nur denkbar sei, wenn es eine rationale Grundlage, die von allen Menschen geteilt werde, gebe. Erkenntnis sei immer intersubjektiv oder zumindest intersubjektivierbar, wenn man von der Erkenntnis aller „normaler”, das heißt sich auf empirische Thesen stützender Menschen ausgeht (Zittel 2007: 449):

Normale Menschen sind unter gegebenen Wahrnehmungsbedingungen im allgemeinen geneigt, die gleichen Behauptungen anzuerkennen. Für die Objektivität empirischer Sätze ist es nicht wesentlich, welche Eindrücke diese Menschen haben, was sie erleben, wenn sie diese Sätze anerkennen. [...] Darum auch scheint mir die vor Kurzem in Przeglad Filozoficzny [Philosophische Rundschau] von Dr. Fleck formulierte Ansicht falsch zu sein, daß es nur eine Sache des "Stils” des Denkens sei, ob man ein Empiriker oder ein Mystiker ist, und daß kein Stil von der Erkenntnistheorie bevorzugt werden dürfe. [...] Der Mensch würde gar nichts von der Welt, in der er lebt, wissen, er würde gar jämmerlich untergehen, wenn er aufhören würde, mit den empirischen Thesen konsequent zu rechnen. (Dąmbska 1937 [Fleck 2011]: 315)

Fleck greift in seiner Antwort auf ein Beispiel aus der Ethnologie zurück:

Aller Aberglaube, Zauberei, alles Wissen der vergangenen Jahrhunderte, wie z.B. die Astrologie und die Alchemie, die ganze mittelalterliche Medizin und schließlich das für uns phantastisch bizarre Wissen der primitiven Völker - all diese Anschauungen haben ihre „Beweise”, die aus eingetroffenen Vorhersagen und erklärten Enttäuschungen hervorgehen, nicht anders als bei unseren Naturwissenschaften. Sie alle scheinen ihren Anhängern anwendbar zu sein - wer würde sich sonst zu ihnen bekennen? Ein afrikanischer Urwaldbewohner „geht” überhaupt nicht „erbärmlich unter”, obwohl keine Rede davon sein kann, daß er in unserem Sinne die „empirischen Thesen” konsequent berücksichtigt. (Fleck 2011 [1937]: 323)

Fleck beharrt hier auf seiner kulturrelativistischen Position: Auch der „wilde” Mensch sei einem Denkzwang und einem Denkkollektiv unterworfen und die für uns erkennbare Diskrepanz in seinem Denken resultiere aus dem anderen Denkstil. Um diesem Denkstil gerecht werden zu können, müssten - ähnlich wie bei Malinowski - auch die kulturellen Prozesse mitberücksichtigt werden. Malinowski unterschied jedoch noch zwischen primitiven und zivilisatorischen Kulturen. Auch Dąmbska vertritt diese Position und wirft Fleck einen Relativismus vor, doch Fleck antwortet ihr:

Das sind weder Sophismen, noch geht damit eine Relativierung der Wahrheit einher. Ich behaupte nicht, daß das alchemistische Gold das echte Gold in unserem Sinne war. Ich behaupte, daß die Alchemisten Gold und überhaupt die materiellen Elemente anders begriffen haben als wir. Sie suchten nicht unser Gold; unsere Elemente wurden erst später eine nützliche Konzeption. Ich behaupte nicht, daß eine Negertrommel ein besseres, sondern ein genauso gutes Kommunikationsmittel auf Entfernung ist wie der drahtlose Telegraph; ich behaupte jedoch, daß das, was ein Neger von dem ihn elektrisierenden Rhythmus der Trommel unmittelbar erfährt, nicht durch eine radiotelegraphische Depesche ersetzt werden kann. (Fleck 2011 [1937]: 325) 
Da alles nun Fleck zufolge denkstilabhängig ist, ist somit eine neutrale Erkenntnis beziehungsweise eine psychologie- und kulturfreie Wissenschaft nicht möglich.

Eine weitere Kontroverse um den Denkstil-Begriff führt Fleck in Przeglad Wspótczesny [Zeitgenössische Rundschau] mit dem Medizinhistoriker und Psychiater Tadeusz Bilikiewicz (1901-1980). Dieser vertritt die Meinung, es gebe doch Feststellungen, die unabhängig vom Denkstil gemacht werden können, nur Geisteskranke seien von solchen Urteilen befreit. Man könne beispielsweise objektiv behaupten, daß ,eine normale menschliche Hand fünf Finger hat" (Bilikiewicz 1939 [Fleck 2011]: 348). Dies sei nämlich keine Frage des Stils, sondern der Betrachtung. Um Bilikiewiczs Argument zu kontern, sucht Fleck nach einem ethnologischen Beispiel, das seine Theorie stützt, und er wird offenbar bei Lévy-Bruhl fündig. In dessen oben genannten Buch Das Denken der Naturvölker heißt es:

Mit den Händen reden, das ist buchstäblich in gewissen Maße mit den Händen denken. [...] In einer großen Zahl niedriger Gesellschaften (Australien, Südamerika etc.) gibt es nur für die Zahlen 1, 2 und manchmal 3 Namen, darüber hinaus sagen die Eingeborenen: „viele, eine Menge, eine große Zahl”. [...] Auf den Andamaninseln sind Zahlworte trotz des außerordentlichen Reichtums der Sprache lediglich für 1 und 2 da, drei wird so umschrieben „eins mehr”, vier ,einige mehr", fünf ,alle". [...] Dann setzt man mit der anderen Hand fort, woraufhin die beiden Hände gefaltet werden, um die Summe von $5+5$ anzudeuten und zugleich die Rechnung durch das Wort adura (alle) als beendet zu erklären. (Lévy-Bruhl 1926: 137, 156 f.)

Fleck schmiedet daraus ein Argument und antwortet Bilikiewicz:

Zahlreiche primitive Gesellschaften (Australien, Südamerika) besitzen nur für die Zahlen 1, 2, 3 separate Namen, ansonsten sagen die Einheimischen: zahlreich, eine Menge, eine große Zahl. Der Satz, daß ,eine Hand fünf Finger hat”, läßt sich also nicht in ihre Sprache übersetzen. Sie sagen: „Eine Hand hat viele Finger”, und das ist eine andere Theorie als die unsere. Andere primitive Kollektive (Papua) verwenden für die Bezeichnung der Zahl fünf das Wort „Hand”, für zehn „zwei Hände”. Sie können also den Satz „Die Hand hat fünf Finger” nicht ohne Tautologie aussprechen, und die Frage, wie viele Finger eine Hand hat, ist für sie praktisch unsinnig, ähnlich wie für uns die Frage: Wie viel kostet ein Zloty? [...] Die Zwergmenschen von den Andamanen sagen statt fünf ,alles”, ihr Satz lautet daher: „Die Hand hat alle Finger”. Dies ist also wieder eine andere Theorie als die unsere. [...]. Dies sind nicht nur sprachliche Unterschiede, denn die Worte, die verwendet werden, um die Zahl „fünf” zu bezeichnen - „eine Menge”, „eine Hand”, ,alles" -, haben gerade einen ganz anderen Umfang als das Wort "fünf”. Das ist ein ganz anderer Denkstil. (Fleck 2011 [1939]: 356)

Nach dem zweiten Weltkrieg werden diese kulturalistischen Begriffsrelativierungen noch um Beispiele aus dem Bereich des Gestaltsehens ergänzt. In seinem Text „Schauen, Sehen, Wissen” erklärt Fleck, daß der Urmensch einen keuchenden Drachen sehe, wenn er zum ersten Mal eine Eisenbahn erblicke, 




Abb. 1 „Ein Urmensch sähe einen keuchenden Drachen” (Fleck 2011 [1947]: 401)/ Bronisław Wojciech Linke': Der Wilde und die Bahn (1947)

das heißt er identifiziere das unbekannte Objekt mit einem Begriff aus der eigenen Denkwelt (Abb. 1):

Wir Heutigen sehen sofort einen Bahnhof, eine Gestalt, die der Urmensch nicht sehen konnte: Er würde auf unzähliges Eisen in verwirrenden Leisten schauen, befestigt auf der Erde, auf Häuschen auf Rädern, auf ein keuchendes Ungeheuer, aus dem Feuer und Rauch herausschlägt, und er sähe wahrscheinlich seine Gestalten: einen Drachen, einen Teufel, wer weiß schließlich, was er sähe, aber nicht unsere gute alte Bahn. (Fleck 2011 [1947]: 400)

Bilder sind Resultate denkstilabhängigen gestalthaften Sehens. Fleck bezeichnet sie deshalb mit dem Ausdruck „Ideogramme”: „graphische Darstellungen gewisser Ideen, gewissen Sinnes, einer Art des Begreifens" (Fleck 1980 [1935]: 183). Auch diesen Terminus übernimmt er von Lévy-Bruhl, der Ideogramme als visuell-motorische Assoziationen, Schemata sowie Modelle definiert (Lévy-Bruhl 1926: 137 f.) und damit ihre automatische Funktion im Wahrnehmungsprozess hervorhebt. Solche Ideogramme als Ausdrücke „gefühlsbetonter Symbolik” (Fleck 1980 [1935]: 179) beobachtet Fleck auch in den anatomischen Bildern. Vesalius' Skelettfiguren (Abb. 2 und 3) symbolisieren beispielsweise mit ihren Requisiten, wie Spaten oder Sense, sowie ihrer pathetischen, leidvollen Haltung den Tod. Sudhoffs Darstellung der Windungen eines Darms sieht wiederum aufgrund vieler Kräuselungen 


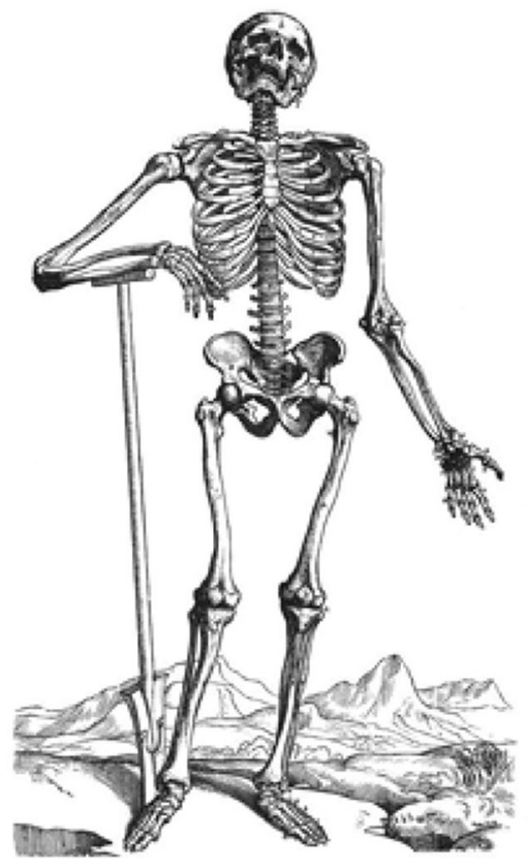

Abb. 2 Die Vesalsch'en Skelettfiguren nach Roth (Fleck 1980 [1935]: 180)

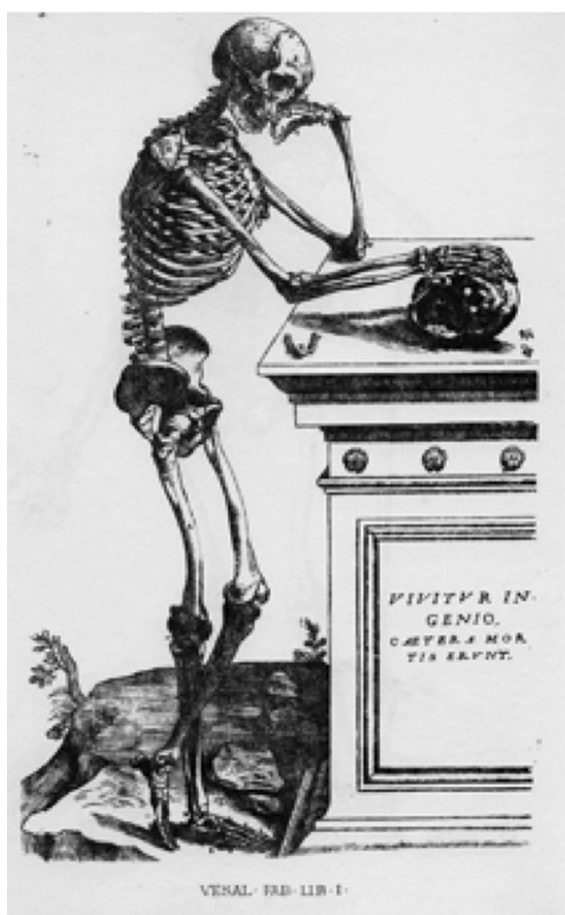

Abb. 3 Die Vesalsch'en Skelettfiguren nach Roth (Fleck 1980 [1935]: 180) 




Abb. 4 Aderlassfigur nach Sudhoff (Fleck 1980 [1935]: 182)

wie eine Schnecke aus (Abb. 4). Doch man könnte Fleck zufolge auch in heutigen anatomischen Bildern ihre besondere intellektuelle Stimmung erkennen. Auch unsere modernen Bilder sind Sinnbilder: „Es gibt kein anderes Sehen als Sinn-Sehen und keine anderen Abbildungen als die SinnAbbildungen" (ebd.: 186).

Resümierend lässt sich sagen, dass Fleck das kulturalistische Wissen aus der Ethnologie beziehungsweise Wahrnehmungstheorie aufgreift, um die Formen der „modernen” Wissenschaft als auf Glauben und magischen Praktiken aufgebaute und von kollektiven Zwängen und Stimmungen determinierte Denkstile anschaulich zu beschreiben. Die Beschäftigung mit fremden Kulturen führe zwar nicht zum Verstehen der anderen Denkstile, denn das sei Fleck zufolge kaum möglich, doch die Konfrontation mit der Fremdheit der anderen Denkart führe zur Prüfung des eigenen Denkstils und schließlich zur Einsicht, dass er auch unentwirrbar kulturabhängig sei. Die eigene Position werde dabei als relativ erkannt und der Verabsolutierung von Standpunkten der Boden entzogen.

\section{Anmerkungen}

1 Frostig gelangt von einem anderen Ausgangspunkt als Fleck zu einer relativistischen Wirklichkeitsauffassung. Zunächst gibt es bei ihm die linguistische Grundannahme, dass Sprache als kollektiv strukturiertes Kommunikationssystem zu verstehen sei. Die mit 
einem Wort bezeichnete Struktur eines Gegenstands könne nur dann von den anderen aufgefasst werden, wenn sie eine kollektive Geltung hat beziehungsweise kollektiv fundiert wurde. Auch dann, wenn das gesprochene Wort keine „reinen Merkmale” (Frostig 1929: 19) enthält, das heißt, wenn beispielsweise der Ton oder Laut bei der Aussprache verfälscht wird, wird es immer auf den im Kollektivbestand vorhandenen Gegenstand zurückgeführt. Einen ähnlichen Mechanismus, in dem die unklaren, gar fehlenden Elemente denkstilgemäß vervollständigt werden, beobachtet später Fleck im Erkenntnisprozess von wissenschaftlichen Phänomenen. Er nennt ihn eine „Transposition” und bedient sich hierbei eines Begriffs aus der Gestalttheorie.

2 „Zu den Strukturen der Gegenstände gelangen wir auf dem Wege einer gerichteten Erkenntnis. [...] In der Erkenntnis der naiven Denkweise ist der Glaube versteckt, daß der erkannte objektive Sachverhalt auch ,wirklich' so ist, wie er erkannt wurde; der naive Verstand schreibt seinen Erkenntnissen einen absoluten Wert zu. Der empirische Gedankenweg muß aber von den absoluten Wahrheiten absehen. [...] Ja er könnte sich eine absolute Erkenntnis gar nicht denken" (Frostig 1929: 21: Fußnote 1). Vgl. dazu den Kommentar in Fleck 2011 [1929]: 66, En IV.

3 ,Just because no idea and no object can exist in isolation from Its cultural context, it is impossible to sever mechanically an item from one culture and place it in another", vgl. Malinowski 1926: 179.

4 „The inner constraint is the result of the gradual training of the organism within a definite set of cultural conditions", vgl. Malinowski 1931: 623.

5 Karl-Heinz Kohl wies mich darauf hin, dass Lévy-Bruhl seine ursprünglichen Ideen in seinen posthum erschienenen Carnets widerrief, vgl. Evans-Pritchard 1981: 122.

6 Der Grafiker Bronisław Wojciech Linke (1906-1962) war Vertreter des metaphorischen Realismus, einer Kunstrichtung, die auf eine anschauliche, oft wörtliche Darstellung literarischer Metaphern zielte. Bekannt wurde er durch seine Hitler-Karikaturen und Antikriegsplakate, insbesondere durch den 1946 entstandenen Zyklus Die Steine schreien, der die Stadt Warschau in Schutt und Asche zeigt. Auch diese Grafiken Linkes könnte man als Illustrationen zu Flecks Texten lesen, wenn er zum Beispiel 1947 in „Schauen, Sehen, Wissen” schreibt: „Es ist klar, daß nur ein Mensch aus unserer Gesellschaft ein ,Haus' sieht, d.h. diese Gestalt aus der ganzen Skala ihrer möglichen Transpositionen heraus erkennt. Heute ist eine Situation möglich, in der ein Bewohner Warschaus ein Haus sieht, aber ein Bewohner New Yorks eine Ruine, einen Haufen Schutt." (Fleck 2011 [1947]: 399).

\section{Literatur}

Bilikiewicz, Tadeusz, [1939] 2011. Bemerkung zum Artikel von Ludwik Fleck „Wissenschaft und Umwelt". In: Sylwia Werner und Claus Zittel, Hg., Ludwik Fleck. Denkstile und Tatsachen. Gesammelte Schriften und Zeugnisse. Berlin: Suhrkamp, 340-352.

Bohr, Nils, 1928. Das Quantenpostulat und die neuere Entwicklung der Atomistik. Die Naturwissenschaften, 16 (15), 245-257.

Dąmbska, Izydora, [1937] 2011. Ist die intersubjektive Ähnlichkeit der Sinneseindrücke eine unentbehrliche Voraussetzung der Naturwissenschaften? In: Sylwia Werner und Claus Zittel, Hg., Ludwik Fleck. Denkstile und Tatsachen. Gesammelte Schriften und Zeugnisse. Berlin: Suhrkamp, 310-319.

Dembowski, Jan, 1927. O poezji nauki [Über die Poesie der Wissenschaft]. In: Ders., Szkice bilogiczne [Biologische Skizzen]. Lemberg: Państwowe Wydawnictwo Książek Szkolnych, 1-6.

Dembowski, Jan, 1946. Psychologia zwierzat. Warschau: Czytelnik [dt.: 1955. Tierpsychologie. Berlin: Akademie-Verlag].

Durkheim, Émile, [1898] 1976. Individuelle und kollektive Vorstellungen. In: Ders., Soziologie und Philosophie. Frankfurt a. M.: Suhrkamp, 45-83.

Egloff, Rainer, 2007. Leidenschaft und Beziehungsproblem: Ludwik Fleck und die Soziologie. In: Bożena Chołuj und Jan C. Joerden, Hg., Von der wissenschaftlichen Tatsache zur 
Wissensproduktion: Ludwik Fleck und seine Bedeutung für die Wissenschaft und Praxis. Frankfurt a. M.: Peter Lang, 79-93.

Evans-Pritchard, Edward E., [1965] 1981. Theorien über primitive Religionen, Frankfurt a. M.: Suhrkamp.

Fehr, Johannes, 2011. ... und nichts als sie. Eine epistemologische Rapsodie. In: Rainer Egloff und Johannes Fehr, Hg., Vérité, Widerstand, Development: At Work with/Arbeiten mit/Travailler avec Ludwik Fleck, Zürich: Collegium Helveticum, Heft 12, 13-20.

Fleck, Ludwik, [1927] 2011. Über einige spezifische Merkmale des ärztlichen Dankens. In: Sylwia Werner und Claus Zittel, Hg., Ludwik Fleck. Denkstile und Tatsachen. Gesammelte Schriften und Zeugnisse. Berlin: Suhrkamp, 41-51.

Fleck, Ludwik, [1929] 2011. Zur Krise der „Wirklichkeit”. In: Sylwia Werner und Claus Zittel, Hg., Ludwik Fleck. Denkstile und Tatsachen. Gesammelte Schriften und Zeugnisse. Berlin: Suhrkamp, 52-69.

Fleck, Ludwik [1934] 2011. Wie entstand die Bordet-Wassermann Reaktion und wie entsteht eine wissenschaftliche Entdeckung im allgemeinen? In: Sylwia Werner und Claus Zittel, Hg., Ludwik Fleck. Denkstile und Tatsachen. Gesammelte Schriften und Zeugnisse. Berlin: Suhrkamp, 181-210.

Fleck, Ludwik, [1935] 1980. Entstehung und Entwicklung einer wissenschaftlichen Tatsache. Einführung in die Lehre vom Denkstil und Denkkollektiv, hg. von Lothar Schäfer und Thomas Schnelle. Frankfurt a. M.: Suhrkamp.

Fleck, Ludwik, [1935] 2011. Über die wissenschaftliche Beobachtung und die Wahrnehmung im allgemeinen. In: Sylwia Werner und Claus Zittel, Hg., Ludwik Fleck. Denkstile und Tatsachen. Gesammelte Schriften und Zeugnisse. Berlin: Suhrkamp, 211-238.

Fleck, Ludwik, [1935] 2011. Zur Frage der Grundlagen der medizinischen Erkenntnis. In: Sylwia Werner und Claus Zittel, Hg., Ludwik Fleck. Denkstile und Tatsachen. Gesammelte Schriften und Zeugnisse. Berlin: Suhrkamp, 239-259.

Fleck, Ludwik, [1936] 2011. Das Problem einer Theorie des Erkennens. In: Sylwia Werner und Claus Zittel, Hg., Ludwik Fleck. Denkstile und Tatsachen. Gesammelte Schriften und Zeugnisse. Berlin: Suhrkamp, 260-309.

Fleck, Ludwik, [1937] 2011. In der Angelegenheit des Artikels von Frau Izydora Dąmbska in „Przegląd Filozoficzny” (Jg. 40, Heft III). In: Sylwia Werner und Claus Zittel, Hg., Ludwik Fleck. Denkstile und Tatsachen. Gesammelte Schriften und Zeugnisse. Berlin: Suhrkamp, 320326.

Fleck, Ludwik, [1939] 2011. Antwort auf die Bemerkungen von Tadeusz Bilikiewicz. In: Sylwia Werner und Claus Zittel, Hg., Ludwik Fleck. Denkstile und Tatsachen. Gesammelte Schriften und Zeugnisse. Berlin: Suhrkamp, 353-360.

Fleck, Ludwik, [1946] 2011. Wissenschaftstheoretische Probleme. In: Sylwia Werner und Claus Zittel, Hg., Ludwik Fleck. Denkstile und Tatsachen. Gesammelte Schriften und Zeugnisse. Berlin: Suhrkamp, 369-389.

Fleck, Ludwik, [1947] 2011. Schauen, Sehen, Wissen. In: Sylwia Werner und Claus Zittel, Hg., Ludwik Fleck. Denkstile und Tatsachen. Gesammelte Schriften und Zeugnisse. Berlin: Suhrkamp, 390-418.

Frostig, Jakob, 1929. Das schizophrene Denken. Phänomenologische Studien zum Problem der widersinnigen Sätze. Leipzig: Thieme.

Griesecke, Birgit, 2009. Vergleichende Erkenntnistheorie. Einführende Überlegungen zum Grundkonzept der Fleckschen Methodologie. In: Birgit Griesecke und Erich Otto Graf, Hg., Fleck-Studien. Bd. 1, Berlin: Parerga, 9-62.

Gumplowicz, Ludwig, 1885. Grundriß der Soziologie, Wien: Manz.

Ingarden, Roman, 1937. Wandlungen in der philosophischen Atmosphäre in Polen. Slavische Rundschau, 4, 224-233.

Jerusalem, Wilhelm, 1924. Die soziologische Bedingtheit des Denkens und der Denkformen. In: Max Scheler, Hg., Versuche zu einer Soziologie des Wissens, München: Duncker \& Humblot,182-207.

Lévy-Bruhl, Lucien, [1918] ${ }^{1} 1921 /{ }^{2}$ 1926. Das Denken der Naturvölker, Wien: Braumüller.

Malinowski, Bronisław, [1922] 2007. Argonauten des westlichen Pazifik. Ein Bericht über Unternehmungen und Abenteuer der Eingeborenen in den Inselwelten von MelanesischNeuguinea. Frankfurt a. M.: Dietmar Klotz.

Malinowski, Bronisław, 1926. The Life of Culture. Forum, 76, 178-186. 
Malinowski, Bronisław, 1931. Culture. Encyclopedia of the Social Sciences, 4, 626-645.

Malinowski, Bronisław, 1941. Man's Culture and Man's Behavior. Sigma Xi Quarterly, 29, 170196.

Stadler, Friedrich, 1997. Studien zum Wiener Kreis. Ursprung, Entwicklung und Wirkung des Logischen Empirismus, Frankfurt a. M.: Suhrkamp.

Stagl, Justin, 1993. Malinowskis Paradigma. In: Wolfdietrich Schmied-Kowarzik und Justin Stagl, Hg., Grundlagen der Ethnologie. Beiträge zur gegenwärtigen Theorie-Diskussion, Berlin: Dietrich Reimer, 93-105.

Stocking, George, 1978. Die Geschichtlichkeit der Wilden und die Geschichte der Ethnologie. Geschichte und Gesellschaft, 4, 520-535.

Wegmarshaus, Gert-Rüdiger, 2007. Vom Denkstil zum Paradigma: Zum Schicksal einer unzeitgemäßen Einsicht. In: Bożena Chołuj und Jan C. Joerden, Hg., Von der wissenschaftlichen Tatsache zur Wissensproduktion. Ludwik Fleck und seine Bedeutung für die Wissenschaft und Praxis, Frankfurt a. M.: Peter Lang, 49-77.

Werner, Sylwia, 2011. Ludwik Fleck und die Wissenskultur der Lemberger Moderne. In: Rainer Egloff und Johannes Fehr, Hg., Vérité, Widerstand, Development: At Work with/Arbeiten mit/ Travailler avec Ludwik Fleck, Zürich: Collegium Helveticum, Heft 12, 59-66.

Zittel, Claus, 2007. Die Entstehung von Entwicklung von Ludwik Flecks „,vergleichender Erkenntnistheorie. In: Bożena Chołuj und Jan C. Joerden, Hg., Von der wissenschaftlichen Tatsache zur Wissensproduktion: Ludwik Fleck und seine Bedeutung für die Wissenschaft und Praxis. Frankfurt a. M.: Peter Lang, 439-466.

Zittel, Claus, 2011. Ludwik Fleck und der Stilbegriff in den Naturwissenschaften. Stil als wissenschaftshistorische, epistemologische und ästhetische Kategorie. In: Horst Bredekamp und John Krois, Hg., Sehen und Handeln, Berlin: Akademie Verlag, 171-206.

\section{Sylwia Werner}

Exzellenzcluster „,Kulturelle Grundlagen von

Integration"/Fachbereich Literaturwissenschaft

Universität Konstanz

78457 Konstanz

Deutschland

E-Mail: sylwia.werner@uni-konstanz.de 